\title{
A human monoclonal antibody derived from a vaccinated volunteer recognizes heterosubtypically a novel epitope on the hemagglutinin globular head of $\mathrm{H1}$ and
}

\section{H9 influenza A viruses}

Naphatsawan Boonsathorn ${ }^{\mathrm{a}, \mathrm{h}}$, Sumolrat Panthong ${ }^{\mathrm{a}, \mathrm{h}}$, Sarawut Koksunan ${ }^{\mathrm{a}}$, Malinee Chittaganpitch $^{\mathrm{b}}$, Siripaporn Phuygun $^{\mathrm{b}}$, Sunthareeya Waicharoen $^{\mathrm{b}}$, Apichai Prachasupap $^{\mathrm{a}, \mathrm{h}}$, Tadahiro Sasaki ${ }^{\mathrm{c}, \mathrm{h}}$, Ritsuko Kubota-Koketsu ${ }^{\mathrm{d}}$, Mayo Yasugi ${ }^{\mathrm{e}}$, Ken-ichiro Ono ${ }^{\mathrm{f}, \mathrm{h}}$, Yasuha Arai $^{\mathrm{c}}$, Takeshi $\mathrm{Kurosu}^{\mathrm{c}, \mathrm{h}}$, Pathom Sawanpanyalert ${ }^{\mathrm{g}}$, Kazuyoshi Ikuta ${ }^{\mathrm{c}, \mathrm{h}, *}$, Yohei Watanabe $\mathrm{c}^{\mathrm{chh}, *}$

${ }^{\text {a }}$ Medical Life Sciences Institute, Department of Medical Sciences, Ministry of Public Health, Muang, Nonthaburi, Thailand, ${ }^{\mathbf{b}}$ National Institute of Health, Department of Medical Sciences, Ministry of Public Health, Muang, Nonthaburi, Thailand, c Department of Virology, Research Institute for Microbial Diseases, Osaka University, Suita, Osaka, Japan, ${ }^{\mathbf{d}}$ Kanonji Institute, The Research Foundation for Microbial Diseases of Osaka University, Kanonji, Kagawa, Japan, ${ }^{\mathbf{e}}$ Graduate School of Life and Environmental Sciences, Osaka Prefecture University, Izumisano, Osaka, Japan, ${ }^{\mathbf{f}}$ Ina Laboratory, Medical \& Biological Laboratories Corporation, Ltd., Ina, Nagano, Japan, 


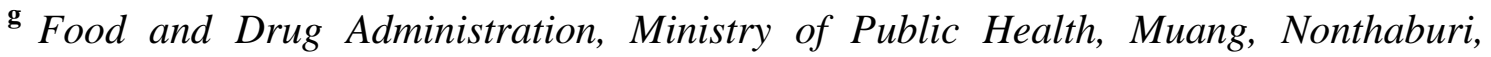
Thailand, $\mathbf{h}$ Japan Science and Technology Agency/Japan International Cooperation Agency, Science and Technology Research Partnership for Sustainable Development (JST/JICA, SATREPS), Tokyo, Japan

* Corresponding author:

\section{Yohei Watanabe and Kazuyoshi Ikuta}

Department of Virology, Research Institute for Microbial Diseases, Osaka University, 3-1 Yamadaoka, Suita, Osaka, Japan; Phone: +81-6-6879-8308

E-mail: nabe@biken.osaka-u.ac.jp (YW); Ikuta@biken.osaka-u.ac.jp (KI) 


\section{Abstract}

Most neutralizing antibodies elicited during influenza virus infection or by vaccination have a narrow spectrum because they usually target variable epitopes in the globular head region of hemagglutinin (HA). In this study, we describe a human monoclonal antibody (HuMAb), 5D7, that was prepared from the peripheral blood lymphocytes of a vaccinated volunteer using the fusion method. The HuMAb heterosubtypically neutralizes group 1 influenza A viruses, including seasonal H1N1, 2009 pandemic H1N1 (H1N1pdm) and avian H9N2, with a strong hemagglutinin inhibition activity. Selection of an escape mutant showed that the HuMAb targets a novel conformational epitope that is located in the HA head region but is distinct from the receptor binding site. Furthermore, Phe114Ile substitution in the epitope made the HA unrecognizable by the HuMAb. Amino acid residues in the predicted epitope region are also highly conserved in the HAs of H1N1 and H9N2. The HuMAb reported here may be a potential candidate for the development of therapeutic/prophylactic antibodies against $\mathrm{H} 1$ and $\mathrm{H9}$ influenza viruses. 
Keywords; Influenza A virus; Human monoclonal antibody; Heterosubtypic neutralizing antibody; Conserved epitope in HA head region

\section{Introduction}

Over the past and current centuries, four human influenza pandemics have emerged (1918 H1N1 Spanish flu, 1952 H2N2 Asian flu, 1968 H3N2 Hong Kong flu and 2009 H1N1pdm) [1]. In all the cases, the hemagglutinin (HA), one of the major antigens on the virion surface, had acquired antigenicity distinct from the HAs of human viruses that circulated at the time. The pandemic viruses developed subsequently to seasonal viruses in humans, imposing huge public health burdens.

HA is classified into subtypes, based on its antigenic properties [2]. To date, 18 HA subtypes have been described. The HAs are also divided into two phylogenetic groups: group 1 (H1, H2, H5, H6, H8, H9, H11, H12, H13, H16, H17 and H18) and group 2 (H3, H4, H7, H10, H14 and H15). HA consists of a head region and stem region, which are genetically and antigenically variable and more conserved, respectively [3]. The HA globular head region includes a receptor binding site (RBS) that mediates viral attachment to target cells through interactions with sialic acid 
receptors, whilst the HA stem region has a fusion domain that induces virus-host membrane fusion, allowing the release of the viral genome into the cytoplasm $[1,4]$. The immune system produces a repertoire of antibodies that mainly target the HA globular head and thus block the virus binding to host cells. Five antigenic sites have been characterized in the $\mathrm{H} 1$ and $\mathrm{H} 3$ globular head region and are designed either as $\mathrm{Sa}$, Sb, $\mathrm{Ca} 1, \mathrm{Ca} 2$ and $\mathrm{Cb}$ or as sites A through $\mathrm{E}[5,6]$. However, these antibodies are usually specific to a particular virus strain or closely related virus strains within one subtype. Furthermore, the HA head region rapidly and continuously accumulates amino acid substitutions to escape from the antibody response. Recently, several anti-stem region antibodies with a broad spectrum against influenza viruses have been reported $[7,8,9,10]$. However, information about conserved HA epitopes is still limited.

Vaccination is considered a promising control measure against pandemic and epidemic influenza but has several limitations, mainly because of viral antigenic drift [11]. Furthermore, the emergence of resistance to antiviral drugs in recent years limits the options available for the control of influenza [12]. Under such circumstances, the interest in human monoclonal antibodies (HuMAbs) as alternative prophylactic and therapeutic agents has been strengthened by the recent identification of heretrosubtypic neutralizing HuMAbs to influenza viruses. Most of these HuMAbs have been derived 
from phage display libraries and found to target the HA stem region [7,10,13,14]. However, HuMAbs generated by this method are the products of random recombination between the immunoglobulin variable region heavy (VH) chain and light (VL) chain genes.

In contrast, we have reported a cell-to-cell fusion method using a new fusion partner cell line, SPYMEG, and donor-derived peripheral blood mononuclear cells (PBMCs) $[9,15,16,17,18]$. In this study, we report the characterization of an anti-influenza HuMAb generated from the PBMCs of a volunteer vaccinated with seasonal influenza vaccine. This antibody, designated 5D7, neutralized heterosubtypically seasonal H1N1, H1N1pdm and H9N2 influenza viruses and binds to a novel epitope, distant from the RBS in the HA globular head.

\section{Materials and methods}

\subsection{Ethics statement}

The study protocol was approved by the ethical committee of the Department of Medical Sciences, Ministry of Public Health, Thailand (approval number TMEC 11-020) and all blood samples were collected after obtaining informed consent. 


\subsection{Viruses}

Several strains of influenza A and B viruses were used in this study: A/New Caledonia/20/1999, A/Brisbane/59/2007 and A/TAK/261/2008 are seasonal H1N1 strains that were prevalent before 2009; A/Suita/1/2009, A/Ayuttaya/568/2009, A/Ayuttaya/610/2009, $\quad$ A/Pathumtani/460/2010, $\quad$ A/Songkhla/19/2011, A/Songkhla/22/2011, A/Chanthaburi/32/2011, A/Chanthaburi/58/2011, A/Surat Thani/41/2011 and A/Nakhon Ratchasima/103/2012 are H1N1pdm strains; A/Chanthaburi/244/2011 is an H3N2 strain; A/Okuda/1957 is an H2N2 strain; A/Turkey/Wisconsin/1/1966 is an H9N2 strain; A/Laos-Nong Khai/286/2007 is an H5N1 strain; B/Malaysia/2506/2004 is an influenza B virus strain. The Thai and Laos strains were kindly provided by the Thai National Influenza Center, National Institute of Health, Thailand, and the other strains were kindly provided by the Research Institute of Microbial Diseases, Osaka University, Japan. Viruses were cultured either in Madin-Darby canine kidney MDCK cells or in 9-days-old embryonated hens' eggs. Virus titers were assayed as focus-forming units (FFU) using a focus-forming assay as previously described [19]. 


\subsection{Fusion partner cell line}

SPYMEG cells (Medical \& Biological Laboratories), which were established by fusing the mouse myeloma cell line SP2/0-Ag 14 and human megakaryoblastic cell line MEG-01 (non-secretors of mouse and human immunoglobulins), were used as fusion partner cells.

\subsection{HuMAb preparation}

Hybridoma cells producing HuMAbs against influenza viruses were prepared using SPYMMEG cells, as previously described [15,18]. Briefly, $10 \mathrm{ml}$ of blood was collected from a healthy volunteer (23 years old, male, Thai) vaccinated in January 2011 with the trivalent HA split influenza vaccine (VAXIGRIP, Sanofi Pasteur), containing antigens from influenza A H1N1 virus, H3N2 virus and influenza B virus. PBMCs were isolated by Ficoll-Plaque Plus (GE healthcare) and then fused with SPYMEG cells using polyethylene glycol 1500 (Roche Diagnostics). Hybridoma cells were selected using the hypoxanthine-aminopterin-thymidine (HAT) selection method in 96-well plates. After 10-14 days culturing, the first round screening for antibodies 
specific for influenza virus was performed by immunofluorescence assay (IFA). The cells in the positive wells were subcloned by limiting dilution and then subjected to the second round of screening by IFA. The hybridoma clones were then cultured and expanded in Hybridoma-SFM (Invitrogen). HuMAb was purified from $100 \mathrm{ml}$ hybridoma culture supernatant by affinity chromatography using HiTrap Protein G HP Columns (GE Healthcare) and then dialyzed against phosphate buffered saline (PBS).

2.5. IFA

IFA was performed as previously described [20], with minor modification. Briefly, infected MDCK cells or transfected human embryonic kidney 293T cells were fixed with 4\% formaldehyde in PBS for 20 min at room temperature (RT) and incubated with hybridoma supernatants or primary antibody for $1 \mathrm{~h}$ at $37^{\circ} \mathrm{C}$, followed by incubation with FITC-conjugated anti-human IgG (for HuMAb) or FITC-conjugated anti-mouse IgG (for mouse MAb) (Jackson ImmunoResearch) for $45 \mathrm{~min}$ at $37^{\circ} \mathrm{C}$. The cells were observed under a fluorescence microscope (Nikon).

\subsection{Immunoglobulin isotyping}


ELISA microplates (MaxiSorp; Nunc) were coated with goat anti-human IgG (Jackson ImmunoResearch) in 0.05 M sodium bicarbonate buffer (pH8.6) overnight at $4^{\circ} \mathrm{C}$. After washing with PBS containing $0.1 \%$ Tween-20 (PBS-T), the wells were blocked with $0.5 \%$ bovine serum albumin (BSA) in PBS at $37^{\circ} \mathrm{C}$ for $1 \mathrm{~h}$. After washing with PBS-T three times, the wells were incubated with hybridoma supernatant at $37^{\circ} \mathrm{C}$ for $2 \mathrm{~h}$. After washing with PBS-T three times, the wells were incubated with secondary HRP-conjugated anti-human IgG1, IgG2, IgG3 or IgG4 (Southern Biotech) at $37^{\circ} \mathrm{C}$ for 1 h. The wells were then washed five times and incubated with 3,3',5,5'-tetramethylbenzidine peroxidase substrate (Kirkegaard \& Perry Laboratories) for 20 min at RT in the dark. The reaction was stopped with $2 \mathrm{~N} \mathrm{H}_{2} \mathrm{SO}_{4}$ solution. The color development was read at $450 \mathrm{~nm}$ in the ELISA Photometer (Biotex ELISA Reader, Biotex).

\subsection{Sequencing of the HuMAb variable regions}

Total RNA was extracted from the hybridoma cells using an RNeasy Mini Kit (Qiagen) and subjected to RT-PCR as described previously [9]. The specific PCR 
product was then sequenced and the sequences obtained were used to search the NCBI database using the IgBLAST website (http://www.ncbi.nlm.nih.gov/igblast/).

\subsection{Virus neutralization (VN) assays}

VN assays were performed as previously described [18]. Briefly, two-fold serial dilutions of purified HuMAb $(100 \mu \mathrm{g} / \mathrm{ml})$ were pre-incubated with $100 \mathrm{FFU}$ of each virus for $1 \mathrm{~h}$ at $37^{\circ} \mathrm{C}$. The mixtures were then inoculated onto MDCK cells in a 96-well plate and incubated at $37^{\circ} \mathrm{C}$ for $1 \mathrm{~h}$. The cells were washed with PBS and then serum free medium was added. After incubation at $37^{\circ} \mathrm{C}$ for $16 \mathrm{~h}$, the cells were fixed and subjected to IFA. The neutralization activity of HuMAb was calculated by the Reed \& Muench method and expressed as the concentration showing 50\% reduction in FFU $\left(\mathrm{VN}_{50}\right)$ compared to the negative control. Each neutralization assay was performed in duplicate.

\subsection{Hemagglutination inhibition (HI) assays}


HI assays were performed using A/Suita/1/2009 as described previously [20] and the reciprocal of the antibody dilution for complete inhibition of hemagglutination was designated as the $\mathrm{HI}$ titer.

\subsection{Construction of expression plasmids}

The HA and viral nucleoprotein (NP) genes of A/Suita/1/2009 and the HA gene of A/New Caledonia/20/1999 were amplified by one step RT-PCR and inserted into the expression vector pCAGGS [18]. Mutant A/Suita/1/2009 HA genes were generated by site-directed mutagenesis PCR (KOD-Plus; Toyobo). The expression plasmids were then transfected into 293T-cells using Transit-LT1 (Mirus) according to the manufacturer's instructions.

\subsection{Western blotting (WB)}

MDCK cells infected with A/Chanthaburi/58/2011 or 293T cells transfected with the A/New Caledonia/20/1999 HA expression plasmid were suspended in loading 
buffer with or without $\beta$-mercaptoethanol and subjected to electrophoresis, followed by western blotting as described previously [21].

\subsection{Selection of escape mutants}

Escape mutants were isolated by culturing A/Suita/1/2009 in the presence of HuMAb, as described previously [17], with minor modifications. Briefly, the virus was incubated with 10-fold diluted HuMAb (to give final concentrations of 5, 0.5 and 0.05 $\mu \mathrm{g} / \mathrm{ml}$ ) at $37^{\circ} \mathrm{C}$ for $1 \mathrm{~h}$. MDCK cells in 24-well plates were then inoculated with the mixtures and cultured with DMEM/F12+GlutaMAX-1 (Invitrogen) supplemented with $0.4 \%$ BSA, $2 \mu \mathrm{g} / \mathrm{ml}$ acetylated trypsin and antibiotics. After culturing for $72 \mathrm{~h}$, the supernatants in each well were collected and subjected to VN and HI assays. The viral samples that showed more than 4-fold reduction of $\mathrm{VN}_{50}$ and $\mathrm{HI}$ titer were subjected to direct sequencing analysis of the entire HA gene.

\subsection{Sequence population analysis of epitope sequences}


Complete and partial sequences of 2656 HA genes from influenza A virus subtypes H1N1, H2N2, H5N1 and H9N2, which were isolated from 2007 to 2013, were obtained from the GISAID database (http://platform.gisaid.org/epi3/frontend). Seasonal H1N1 and H1N1pdm sequences were derived from the viruses isolated in Thailand and Japan. H5N1 and H9N2 sequences were derived from viruses isolated in Asia. H2N2 sequences were derived from the same geographical area. These sequences were aligned using the MAFFT program [22] and used to determine a consensus sequence for each subtype. The prevalence of mutations for each subtype virus was then calculated and compared among subtypes.

\section{Results}

\subsection{Preparation of HuMAb against influenza A viruses}

PBMCs were obtained from a healthy volunteer 7 and 14 days after receiving the trivalent seasonal influenza vaccine. The PBMCs were fused with SPYMEG cells to generate hybridomas. Ultimately, a hybridoma clone designated 5D7, which was derived from the PBMCs collected at 7 days after vaccination, was selected. The 5D7 secreted HuMAb that heterosubtypically cross-reacted with group 1 influenza A viruses, 
including seasonal H1N1, H1N1pdm and H9N2 (Table 1). In contrast, the HuMAb did not react with other group 1 viruses (such as H2N2 and H5N1), group 2 viruses or influenza B viruses. The isotype of the HuMAb was IgG1 and utilized germline VH gene, VH3-23, DH3-22 and JH4. IFA using transfected cells showed that the HuMAb targeted HA (Table 2).

\subsection{Neutralizing ability of the HuMAb}

We performed an in vitro VN assay to evaluate the neutralizing activity of the HuMAb against group 1 influenza A viruses and influenza B viruses. An irrelevant, anti-dengue HuMAb D23-1G7C2 was used as the negative control in this study. In accordance with the reactivity, the HuMAb showed cross-neutralizing activity against seasonal H1N1, H1N1pdm and H9N2 influenza virus strains (Table 1). In particular, the HuMAb showed strong neutralizing activity against H1N1pdm 2009 and 2010 isolates, with $\mathrm{VN}_{50}$ values of $0.70-3.01 \mu \mathrm{g} / \mathrm{ml}$ (Table 1), whilst it neutralized the 2011 and 2012 isolates with $\mathrm{VN}_{50}$ values ranging from 2.65-11.68 $\mu \mathrm{g} / \mathrm{ml}$, which were approximately 3.31-fold higher than $\mathrm{VN}_{50}$ for 2009 and 2010 isolates. Moreover, the HuMAb did not 
neutralize H2N2 and H5N1 strains. D23-1G7C2 showed little neutralizing activity against the influenza viruses.

Next, we tried to clarify the mechanism(s) underlying the neutralizing ability of the HuMAb. An HI assay showed that the HuMAb had HI activity against both seasonal H1N1 and H1N1pdm (Table 1). Western blotting analysis also showed that the HuMAb recognized A/Suita/1/2009 HA under non-reducing conditions but had no such reactivity under reducing conditions (Table 2). In contrast, 5E4, which is a representative HuMAb that recognizes a linear epitope, detected the HA under both non-reducing and reducing conditions. These results suggested that the HuMAb cross-neutralized $\mathrm{H} 1$ and $\mathrm{H} 9$ influenza viruses by preventing the viruses from binding to the cells and that the epitope was conformational in the HA globular head.

\subsection{Mapping the epitope recognized by the HuMAb}

To identify the epitope recognized by the HuMAb, we selected escape mutants of $\mathrm{A} / \mathrm{Suita} / 1 / 2009$ in the presence of increasing concentrations of the HuMAb. A potential escape mutant was obtained when the virus was passaged three times. We confirmed little $\mathrm{HI}$ and neutralizing activity of the HuMAb against the escape mutant, 
even at the concentration of $100 \mu \mathrm{g} / \mathrm{ml}$ (Table 2). The HA nucleotide sequences of the parental virus and the escape mutant were then determined and the deduced amino acid sequences were compared between the two viruses. We found a single amino acid substitution, F114I (H3 numbering), in the HA. A structural study showed that the amino acid residue was involved in the conformation of a distinct structural domain (at positions 112-118) that is located between the antigenic sites $\mathrm{Ca} 1$ and $\mathrm{Cb}$ in the $\mathrm{HA}$ head region (Figure 1A, red region).

To further evaluate the effects of the amino acid substitution, expression plasmids encoding A/Suita/1/2009 HA or the mutant HA with the F114I substitution were constructed. IFA using the transfected cells showed that the HuMAb detected parental A/Suita/1/2009 but had considerably lower reactivity with the F114I mutant HA (Figure 1B, right column). In contrast, C179, which targets the HA stalk region, reacted with both HAs (Figure 1B, middle column). C43, which targets NP, showed no reactivity with the HAs (Figure $1 \mathrm{~B}$, left column). These results suggested that the residue at 114 was critical for binding of the HuMAb to HA and that the domain identified (at positions 112-118) constituted, at least partially, the epitope recognized by the HuMAb. 


\subsection{Sequence variations within the epitope recognized by the HuMAb}

The HA sequences of group 1 influenza viruses (seasonal H1N1, H1N1pdm, H2N2, H5N1 and H9N2) were searched in the GISAID database and amino acid conservation of the regions corresponding to the epitope (positions 112-118 in H1 HA) were analyzed and compared among the subtypes. The result showed that seasonal H1N1, H1N1pdm and H9N2 shared the amino acid residues at positions 112, 113 and 116 (S, S and R, respectively) (Figure 1C). In addition, although H9N2 viruses had tyrosine (Y) at residue 114 instead of phenylalanine (F), we estimated that Y114 had little influence on the interaction between HA and the HuMAb because of the high similarity of these amino acids. This indicated that seasonal H1N1, H1N1pdm and H9N2 viruses have a more similar amino acid motif within the predicted epitope than the other viruses (Figure 1C, residues shown in red), which agreed with the heterosubtypic reactivity and neutralizing activity of the HuMAb to the three subtypes.

In addition, amino acid conservation of the corresponding epitope region of the H1N1 and H9N2 HAs was $>90 \%$ over the past seven years (Table 3), with the exception of relatively moderate conservation at residue 112 of H9N2 HA (56-90.9\%). These results showed that the HA epitope recognized by the HuMAb was highly conserved among H1 and H9 subtype influenza A viruses. 


\section{Discussion}

Several heterosubtypic neutralizing HuMAbs with broad spectrum activity against influenza A viruses have been described recently [7,10,14,23]. Most of the HuMAbs were isolated from phage display libraries and recognized conserved epitopes located in the HA stem region. In contrast, information about conserved epitopes located in the HA globular head region is rather limited. Thus, it is not known whether equivalent HuMAbs that target the globular head region are produced in the course of the immune response to influenza virus. The main finding of this study is that heterosubtypic neutralizing IgG antibodies that target HA globular head can be elicited in humans following exposure to seasonal influenza vaccine.

Although S139/1 was recently reported to neutralize heterosubtypically the H1, H2, H3 and H13 subtypes of influenza A viruses and recognize a conserved epitope in the HA head region [24], S139/1 is a mouse MAb. In contrast, this study for the first time demonstrates that there is a conserved epitope in the HA globular head which is recognized by neutralizing $\operatorname{HuMAb(s)}$ elicited by the immune response to seasonal influenza vaccine. Although we cannot be certain, the HuMAb was likely to be 
produced by memory B cells because the PBMC were collected from an adult volunteer with a history of repeated exposure to influenza viruses, including seasonal H1N1 and H1N1dpm. Indeed, the HuMAb is encoded by a VH gene germline with a high somatic mutation frequency (approximately 20\% at VH3-23, data not shown), implying previous exposure to influenza virus or vaccinations.

Epitope mapping suggested that residues 112 to 118 in the HA globular head region constitute a structural epitope recognized by the HuMAb. The amino acid sequences corresponding to this region are highly conserved among H1 and H9 subtype viruses. A structural analysis also showed that this region is located away from the RBS in the HA head region. Most heterosubtypic cross-neutralizing HuMAb have been reported to recognize epitopes located mainly in the most conserved HA stem region [7,10,14,23]. Also, a few HuMAbs that target relatively conserved epitopes adjacent to or overlapping the RBS in the head region were shown to be subtype-specific $[17,25,26]$. To our knowledge, 5D7 is the first heterosubtypic neutralizing HuMAb that targets a conserved epitope distinct from the RBS in the HA globular head region. The identified novel epitope is located between antigenic sites Ca1 and Cb. Similar epitopes were reported recently by others. An H5N1-specific mouse MAb HA-7 was shown to recognize a structural epitope that consists of two segments, NV/PE at residues 87-79 
and HFEKIW at residues 113-118; the latter overlaps the epitope identified in this study [27]. A HuMAb D1-8, which is derived from human antibody VH and VL repertoires and specific to H3 influenza viruses, was reported to target a conserved epitope between antigenic sites D and E at the globular head of H3 HA [25], which correspond to Ca1 and $\mathrm{Cb}$, respectively, in $\mathrm{H} 1 \mathrm{HA}$.

The amino acid residue at position 114 was critical for the HuMAb binding to the HA globular head. A database search also showed that the amino acid sequences of the epitope region (at positions 112-118) are highly conserved among H1 and H9 influenza viruses. Thus, the HuMAb reported here may be a potential candidate for the future development of effective anti-influenza prophylactic and therapeutic antibodies. In addition, our findings of a novel epitope that is highly conserved in the HA globular head of H1 and H9 influenza viruses should provide useful information for designing and developing a universal vaccine against influenza viruses.

\section{Acknowledgements}

This work was supported by the JST/JICA as part of the Science and Technology Research Partnership for Sustainable Development (SATREPS) 
(08080924). Naphatsawan Boonsathorn is supported by the Japan Society for the Promotion of Science (JSPS) RONPAKU program. We thank Dr. Pannamthip Pitaksajjakul, Faculty of Tropical Medicine, Mahidol University for kindly providing D23-1G7C2 HuMAb.

\section{References}

[1] Y. Watanabe, M.S. Ibrahim, Y. Suzuki, et al., The changing nature of avian influenza A virus (H5N1), Trends Microbiol 20 (2012) 11-20.

[2] R.G. Webster, W.J. Bean, O.T. Gorman, et al., Evolution and ecology of influenza A viruses, Microbiol Rev 56 (1992) 152-179.

[3] N. Pica, P. Palese, Toward a universal influenza virus vaccine: prospects and challenges, Annu Rev Med 64 (2013) 189-202.

[4] J.J. Skehel, D.C. Wiley, Receptor binding and membrane fusion in virus entry: the influenza hemagglutinin, Annu Rev Biochem 69 (2000) 531-569.

[5] A.J. Caton, G.G. Brownlee, J.W. Yewdell, et al., The antigenic structure of the influenza virus A/PR/8/34 hemagglutinin (H1 subtype), Cell 31 (1982) 417-427. 
[6] I.A. Wilson, J.J. Skehel, D.C. Wiley, Structure of the haemagglutinin membrane glycoprotein of influenza virus at 3 A resolution, Nature 289 (1981) 366-373.

[7] D.C. Ekiert, G. Bhabha, M.A. Elsliger, et al., Antibody recognition of a highly conserved influenza virus epitope, Science 324 (2009) 246-251.

[8] Y. Okuno, Y. Isegawa, F. Sasao, et al., A common neutralizing epitope conserved between the hemagglutinins of influenza A virus $\mathrm{H} 1$ and H2 strains, J Virol 67 (1993) 2552-2558.

[9] Y. Pan, T. Sasaki, R. Kubota-Koketsu, et al., Human monoclonal antibodies derived from a patient infected with 2009 pandemic influenza A virus broadly cross-neutralize group 1 influenza viruses, Biochem Biophys Res Commun 450 (2014) 42-48.

[10] J. Sui, W.C. Hwang, S. Perez, et al., Structural and functional bases for broad-spectrum neutralization of avian and human influenza A viruses, Nat Struct Mol Biol 16 (2009) 265-273.

[11] K. Subbarao, Y. Matsuoka, The prospects and challenges of universal vaccines for influenza, Trends Microbiol 21 (2013) 350-358.

[12] M.G. Ison, Antivirals and resistance: influenza virus, Curr Opin Virol 1 (2011) 563-573. 
[13] D. Corti, J. Voss, S.J. Gamblin, et al., A neutralizing antibody selected from plasma cells that binds to group 1 and group 2 influenza A hemagglutinins, Science 333 (2011) 850-856.

[14] D.C. Ekiert, R.H. Friesen, G. Bhabha, et al., A highly conserved neutralizing epitope on group 2 influenza A viruses, Science 333 (2011) 843-850.

[15] R. Kubota-Koketsu, H. Mizuta, M. Oshita, et al., Broad neutralizing human monoclonal antibodies against influenza virus from vaccinated healthy donors, Biochem Biophys Res Commun 387 (2009) 180-185.

[16] T. Sasaki, C. Setthapramote, T. Kurosu, et al., Dengue virus neutralization and antibody-dependent enhancement activities of human monoclonal antibodies derived from dengue patients at acute phase of secondary infection, Antiviral Res 98 (2013) 423-431.

[17] M. Yasugi, R. Kubota-Koketsu, A. Yamashita, et al., Emerging antigenic variants at the antigenic site Sb in pandemic A(H1N1)2009 influenza virus in Japan detected by a human monoclonal antibody, PLoS One 8 (2013) e77892.

[18] M. Yasugi, R. Kubota-Koketsu, A. Yamashita, et al., Human monoclonal antibodies broadly neutralizing against influenza B virus, PLoS Pathog 9 (2013) e1003150. 
[19] Y. Watanabe, M.S. Ibrahim, H.F. Ellakany, et al., Acquisition of human-type receptor binding specificity by new H5N1 influenza virus sublineages during their emergence in birds in Egypt, PLoS Pathog 7 (2011) e1002068.

[20] Y. Watanabe, M.S. Ibrahim, H.F. Ellakany, et al., Antigenic analysis of highly pathogenic avian influenza virus $\mathrm{H} 5 \mathrm{~N} 1$ sublineages co-circulating in Egypt, J Gen Virol 93 (2012) 2215-2226.

[21] Y. Watanabe, N. Ohtaki, Y. Hayashi, et al., Autogenous translational regulation of the Borna disease virus negative control factor $\mathrm{X}$ from polycistronic mRNA using host RNA helicases, PLoS Pathog 5 (2009) e1000654.

[22] K. Katoh, K. Misawa, K. Kuma, et al., MAFFT: a novel method for rapid multiple sequence alignment based on fast Fourier transform, Nucleic Acids Res 30 (2002) 3059-3066.

[23] D. Corti, A.L. Suguitan, Jr., D. Pinna, et al., Heterosubtypic neutralizing antibodies are produced by individuals immunized with a seasonal influenza vaccine, J Clin Invest 120 (2010) 1663-1673.

[24] R. Yoshida, M. Igarashi, H. Ozaki, et al., Cross-protective potential of a novel monoclonal antibody directed against antigenic site B of the hemagglutinin of influenza A viruses, PLoS Pathog 5 (2009) e1000350. 
[25] E. Benjamin, W. Wang, J.M. McAuliffe, et al., A Broadly Neutralizing Human Monoclonal Antibody Directed against a Novel Conserved Epitope on the Influenza Virus H3 Hemagglutinin Globular Head, J Virol 88 (2014) 6743-6750.

[26] Y. Iba, Y. Fujii, N. Ohshima, et al., Conserved Neutralizing Epitope at Globular Head of Hemagglutinin in H3N2 Influenza Viruses, J Virol 88 (2014) 7130-7144.

[27] L. Du, L. Jin, G. Zhao, et al., Identification and structural characterization of a broadly neutralizing antibody targeting a novel conserved epitope on the influenza virus H5N1 hemagglutinin, J Virol 87 (2013) 2215-2225.

\section{Figure legends}

Fig.1. Epitope mapping. (A) Location of the epitope recognized by the HuMAb. The three dimensional structure of the H1N1pdm HA trimer is shown with the locations of H1 HA antigenic sites (Sa, Sb, Ca1, Ca2 and Cb). The novel epitope identified in this study is shown in red. The defined five H1 HA antigenic sites are also shown in other colors. The crystal structure of the HA of A/California/04/2209 (H1N1pdm) (PDB ID 3LZG) was used as a template. (B) Effect of the F114I mutation on the reactivity to 5D7 HuMAb. 293T cells were transfected with a plasmid expressing A/Suita/1/2009 (H1N1) 
HA or the variant HA with the F114I substitution and the reactivity to the HuMAb was determined by IFA. The introduced mutations are on the left and MAbs used for IFA are at the top. (C) Alignment of consensus amino acid sequences that correspond to the epitope region for each subtype (at positions 112-118 at H1 HA). The amino acid motif that is shared by seasonal H1N1, H1N1pdm and H9N2 is shown in red. The numbers of sequences used to determine the consensus sequences are on the right.

Table titles

Table 1. Summary of reactivity, neutralizing activity and HI activity of 5D7 HuMAb.

Table 2. Characterization of 5D7 HuMAb.

Table 3. Conservation of amino acids in the HA epitope of H1N1 and H9N2 influenza viruses. 
Table 1. Summary of reactivity, neutralizing activity and HI activity of 5D7 HuMAb.

\begin{tabular}{|c|c|c|c|c|c|c|c|c|}
\hline \multicolumn{4}{|c|}{ Influenza virus } & \multicolumn{2}{|c|}{ Reactivity (IFA) ${ }^{a}$} & \multicolumn{2}{|c|}{ Neutralizing activity ${ }^{\mathrm{b}}\left(\mathrm{VN}_{50} \mu \mathrm{g} / \mathrm{ml}\right)$} & \multirow{2}{*}{$\begin{array}{l}\text { HI } \text { activity }^{c}(\mu \mathrm{g} / \mathrm{ml}) \\
5 \mathrm{D} 7\end{array}$} \\
\hline Type & Lineage & Subtype & Strain & $5 \mathrm{D} 7$ & $\mathrm{C} 43^{\mathrm{d}}$ & $5 \mathrm{D} 7$ & $\mathrm{D} 23-1 \mathrm{G} 7 \mathrm{C} 2^{\mathrm{e}}$ & \\
\hline \multirow[t]{17}{*}{ A } & Group 1 & Seasonal H1N1 & A/New Caledonia/20/1999 & + & + & $+(2.93)$ & - & nd \\
\hline & & & A/Brisbane/59/2007 & + & + & $+(2.94)$ & - & nd \\
\hline & & & A/TAK/261/2008 & + & + & $+(5.96)$ & - & $+(6.25)$ \\
\hline & & H1N1pdm & A/Suita/1/2009 & + & + & $+(0.70)$ & - & $+(12.50)$ \\
\hline & & & A/Ayuttaya/568/2009 & $\mathrm{nd}^{\mathrm{f}}$ & nd & $+(3.01)$ & - & $+(6.25)$ \\
\hline & & & A/Ayuttaya/610/2009 & nd & nd & $+(1.33)$ & - & $+(3.13)$ \\
\hline & & & A/Pathumtani/460/2010 & nd & nd & $+(1.39)$ & - & nd \\
\hline & & & A/Songkhla/19/2011 & nd & nd & $+(2.65)$ & - & nd \\
\hline & & & A/Songkhla/22/2011 & nd & nd & $+(3.05)$ & - & nd \\
\hline & & & A/Chanthaburi/32/2011 & nd & nd & $+(2.88)$ & - & $+(6.25)$ \\
\hline & & & A/Chanthaburi/58/2011 & + & + & $+(5.91)$ & - & $+(6.25)$ \\
\hline & & & A/Surat Thani/41/2011 & nd & nd & $+(5.79)$ & - & nd \\
\hline & & & A/Nakhon Ratchasima/103/2012 & + & + & $+(11.68)$ & - & $+(12.50)$ \\
\hline & & H9N2 & A/Turkey/Wisconsin/1/1966 & + & + & $+(24.02)$ & - & nd \\
\hline & & $\mathrm{H} 2 \mathrm{~N} 2$ & A/Okuda/1957 & - & + & $-(>100)$ & - & nd \\
\hline & & H5N1 & A/Laos-Nong Khai/286/2007 & - & + & $-(>100)$ & - & nd \\
\hline & Group 2 & H3N2 & A/Chanthaburi/244/2011 & - & + & nd & - & nd \\
\hline B & & & B/Malaysia/2506/2004 & - & - & nd & - & nd \\
\hline
\end{tabular}

${ }^{a}$ MDCK cells infected with the viruses indicated were used for IFA.

${ }^{\mathrm{b}}$ MDCK cells infected with the viruses indicated were used for in vitro VN assay.

${ }^{c}$ Human O-type or goose red blood cells were used for the assays.

${ }^{d}$ C43 is a mouse MAb that broadly cross-reacts with NPs of influenza A viruses.

${ }^{\mathrm{e}} \mathrm{D} 23-1 \mathrm{G} 7 \mathrm{C} 2$ is a HuMAb that nutralizes dengue virus.

${ }^{\mathrm{f}} \mathrm{nd}$, not determined. 


\section{Table 2. Summary of characerization of 5D7 HuMAb}

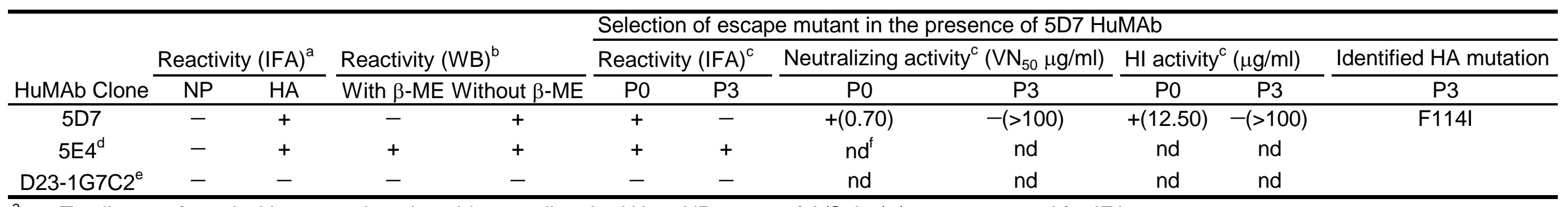

${ }^{a}$ 293T cells transfected with expression plasmids encoding the HA or NP genes of A/Suita/1/2009 were uesd for IFA.

${ }^{\mathrm{b}} \beta$-ME indicates $\beta$-mercaptoethanol.

${ }^{c}$ A/Suita/1/2009 strain was used for the assays. PO and P3 indicate passage numbers.

${ }^{d} 5 E 4$ is a HuMAb that recognizes an linear epitope in HA1 of H1N1pdm.

${ }^{\mathrm{e}} \mathrm{D} 23-1 \mathrm{G} 7 \mathrm{C} 2$ is a HuMAb that recognizes dengue virus.

${ }^{\mathrm{f}} \mathrm{nd}$, not determined. 
Table 3. Conservation of amino acids in the HA epitope of H1N1 and N9N2 influenza viurses.

\begin{tabular}{|c|c|c|c|c|c|c|c|c|c|c|c|c|c|c|}
\hline \multirow[b]{3}{*}{ amino acid type on $\mathrm{H} 1(\mathrm{H} 9) \mathrm{HA}^{\mathrm{d}}$} & \multicolumn{14}{|c|}{$\%$ of strains showing amino acid residue ${ }^{a}$ (no. of strains analyzed) } \\
\hline & \multicolumn{2}{|c|}{ Seasonal $\mathrm{H} 1 \mathrm{~N} 1^{\mathrm{b}}(202)$} & \multicolumn{5}{|c|}{ H1N1pdm ${ }^{b}$ (998) } & \multicolumn{7}{|c|}{$\mathrm{H} 9 \mathrm{~N} 2^{\mathrm{C}}(1110)$} \\
\hline & $2007(63)$ & $2008(139)$ & $2009(135)$ & $2010(389)$ & $2011(315)$ & $2012(28)$ & $2013(131)$ & $2007(177)$ & $2008(132)$ & $2009(292)$ & $2010(252)$ & $2011(39)$ & $2012(100)$ & $2013(118)$ \\
\hline$\overline{\mathrm{S} 112}$ & 100 & 100 & 100 & 100 & 100 & 100 & 100 & 86.4 & $\overline{90.9}$ & 79.1 & 65.4 & 76.9 & 56 & 74.5 \\
\hline S113 & 100 & 100 & 100 & 99.7 & 100 & 100 & 100 & 100 & 100 & 100 & 100 & 100 & 100 & 99.1 \\
\hline$F(Y) 114$ & 100 & 100 & 100 & 100 & 100 & 100 & 100 & 99.4 & 99.2 & 99.6 & 99.6 & 100 & 100 & 100 \\
\hline $\mathrm{E}(\mathrm{Q}) 115$ & 100 & 100 & 100 & 97.1 & 99.6 & 100 & 100 & 94.9 & 93.9 & 89.3 & 95.6 & 100 & 99 & 99.1 \\
\hline R116 & 100 & 100 & 100 & 99.4 & 100 & 100 & 98.4 & 98.3 & 97.7 & 93.8 & 100 & 100 & 90 & 99.1 \\
\hline $\mathrm{F}(\mathrm{I}) 117$ & 100 & 100 & 100 & 100 & 100 & 100 & 100 & 92.6 & 95.4 & 91.7 & 98.4 & 100 & 88 & 99.1 \\
\hline$E(Q) 118$ & 100 & 100 & 100 & 100 & 100 & 100 & 100 & 94.9 & 95.4 & 92.4 & 99.6 & 100 & 100 & 100 \\
\hline
\end{tabular}

${ }^{a}$ HA sequences were collected from GISAID database (http://platform.gisaid.org/).

${ }^{\mathrm{b}} \mathrm{HA}$ sequences of H1N1 influenza viruses detected in Thailand and Japan were extracted from GISAID.

${ }^{c}$ HA sequences of H9N2 influenza viruess detected in Asia were extracted from GISAID.

${ }^{\mathrm{d}} \mathrm{H} 3$ numbering. 
A

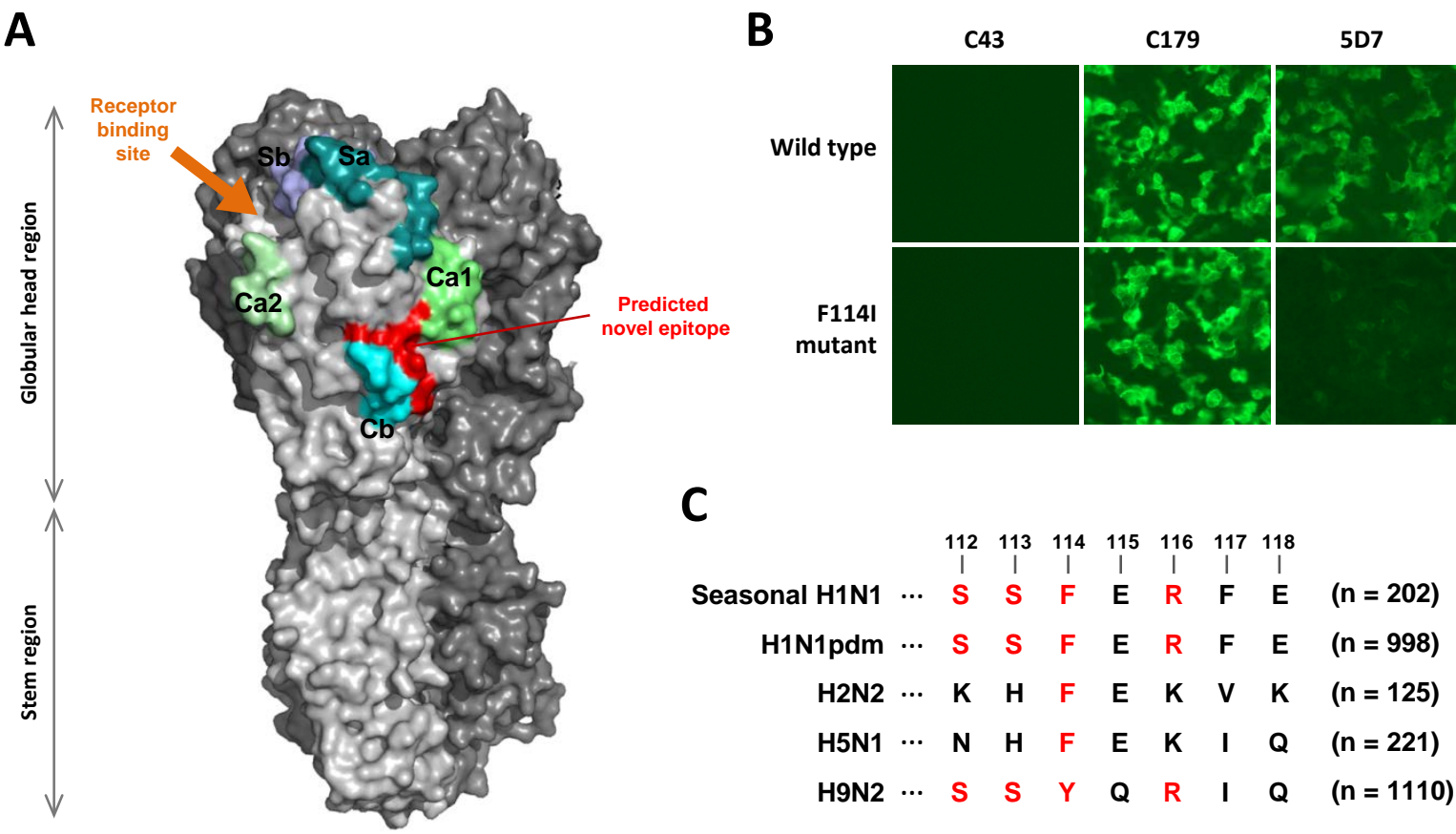

B

C

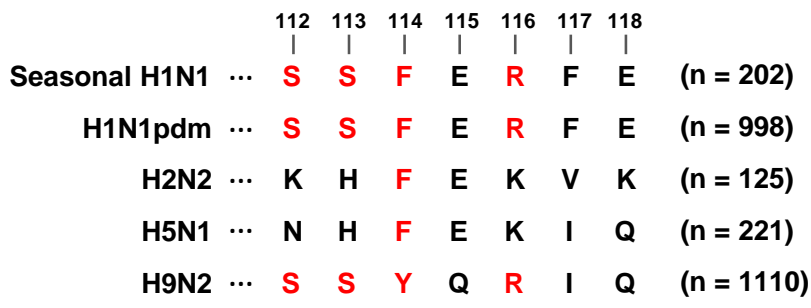

$$
\begin{array}{llllllllll}
\text { H2N2 } & \cdots & \text { K } & \text { H } & \text { F } & \text { E } & \text { K } & \text { V } & \text { K } & (n=125) \\
\text { H5N1 } & \cdots & \text { N } & \text { H } & \text { F } & \text { E } & \text { K } & \text { I } & \text { Q } & (n=221) \\
\text { H9N2 } & \cdots & \text { S } & \text { S } & \text { Y } & \text { Q } & R & \text { I } & \text { Q } & (n=1110)
\end{array}
$$

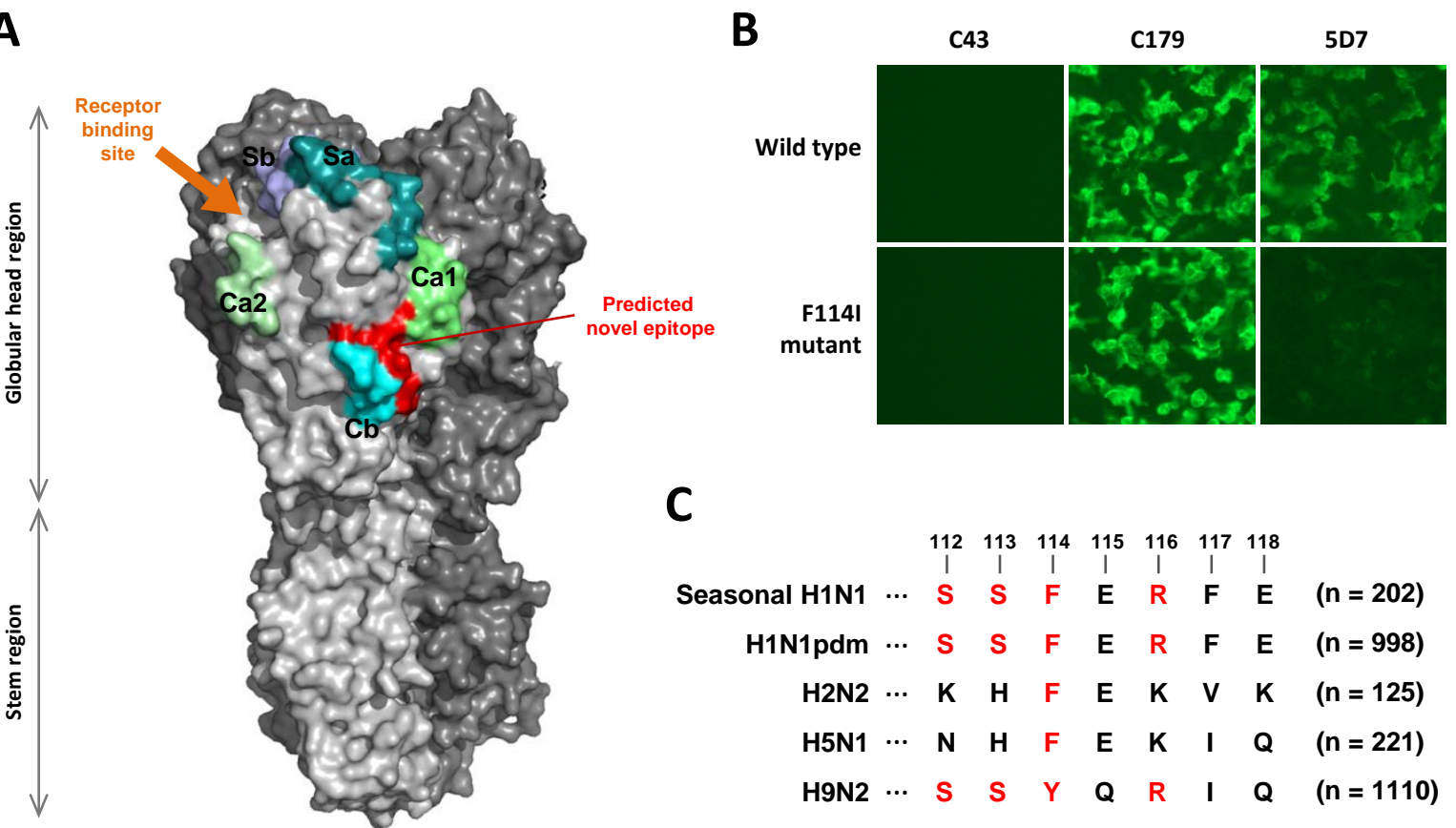

